\title{
EFFICIENT COMPUTATION OF THE REALIZABLE MIMO DFE
}

\author{
Sander Wahls and Holger Boche \\ Technische Universität Berlin \\ Lehrstuhl für Informationstheorie und theoretische Informationstechnik \\ Einsteinufer 25, 10587 Berlin, Germany \\ \{sander.wahls,holger.boche\}@mk.tu-berlin.de
}

\begin{abstract}
Realizable DFEs are DFEs with stable and causal IIR filters and finite decision delay. Computational complexity of current algorithms to compute them usually grows cubically with the decision delay. In this paper, we show how complexity can be reduced to quadratic. We compare two approaches, the so-called polynomial approach and a novel state-space approach using inner-outer factorization. In both cases finite linear equation systems with structure lie at the heart of the realizable DFE. Displacement structure theory allows to solve them efficiently.
\end{abstract}

Index Terms - MIMO systems, Decision feedback equalizers, IIR digital filters, Computation time

\section{INTRODUCTION}

In the literature, numerous approaches to compute decision feedback equalizers (DFEs) for multiple-input multiple-output (MIMO) channels can be found. Among those, the most popular probably are the fast algorithms for the finite impulse response (FIR) DFE, which has a special problem structure that can be exploited in various ways (see e.g. [1], [2]). Here, feed-forward and feed-backward filters (and channel) are constrained to FIR filters. FIR filters are inherently stable, which turns computation of optimal filters into a simple finite linear equation system with structure. Structured linear equation systems can be solved quite efficiently, which is why quadratic complexity in the decision delay can be achieved for the FIR DFE. However, the FIR DFE usually is suboptimal. In fact, it is known that the feed-forward and feed-backward filters of the optimal DFE are simultaneously FIR if and only if the channel is FIR and the noise is white [3, p. 125]. Optimal DFEs employ infinite impulse response (IIR) filters, in general. In order to be physically realizable, the filters must be stable and causal. Various approaches exist to compute optimal realizable DFEs. The polynomial approach has been established in [3]. Other approaches work with state-space models and utilize the Wiener-Kalman filter [4], [5] or optimal control [6]. The authors of [3, p. 127] claim that the complexity of their approach grows cubically with the decision delay. In the other approaches, a virtual MIMO channel is created by modeling the feedback path of the DFE as additional (noisefree) part of the channel. Then, the filters are obtained from the optimal linear equalizer for the virtual channel. However, since the degree of the virtual channel grows linearly with the decision delay, complexity of this operation grows cubically.

In this paper, we make two contributions.

1) We show that the polynomial approach can be solved with quadratic complexity in the decision delay if the channel is square, and

2) we derive a novel state-space approach based on inner-outer factorization which achieves a similar complexity (also in non-square channels).

This work was supported by the German Research Foundation (DFG) under grant BO 1734/11-2.
In turns out that both approaches require some form of coprime factorization (with complexity not depending on the decision delay), and solution of a finite linear equation system with structure. The difference between the inner-outer and the polynomial approach is that the linear equation system in the inner-outer approach is simpler. Therefore, the inner-outer approach can be more efficient. Furthermore, we can carry it out efficiently also for non-square channels. Another difference is that the assumptions on the channel realizations are less restrictive in the inner-outer approach.

Remark 1 (Decision Delay). The decision delay is a major influence on the performance of DFEs (see e.g. [7], [1], [8]). Optimal choice of the decision delay for a FIR DFE is a complicated problem which can only be solved by numerical search, in general [7]. ${ }^{1}$ In contrast, it is simple to see that for IIR DFEs an increased decision delay always increases performance. ${ }^{2}$ This is an advantage of the IIR DFE. Also note that depending on channel and noise characteristics the first decision delay that achieves any close-tooptimal performance may become arbitrary large. Hence, a low complexity in the decision delay is important.

Remark 2 (IIR Channels). The FIR channel model is undoubtedly the predominant model in communications. Nevertheless, recent investigations have shown that IIR channel models may provide much better approximations of a real channel (compared to FIR models) if low-dimensional channel approximations are sought [9]. Most FIR DFE algorithms are designed for FIR channels and cannot cope with IIR channels. (Although this disadvantage can be circumvented using the approach in [9, III.B].) In contrast, the algorithms in this paper naturally support IIR channels.

Remark 3 (Spatial Feedback). Although in MIMO systems decision feedback is also possible in the spatial domain (see e.g. [1]), we only consider feedback in the temporal domain in this paper.

Notation: The Frobenius norm of a complex matrix is

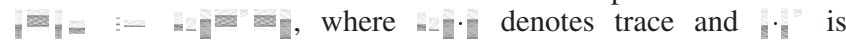
conjugate transpose. We denote the space of causal and stable rational matrices by _ ( or _ _ when the dimensions are not obvious), and write of a rational matrix $\ldots$. The norm of is

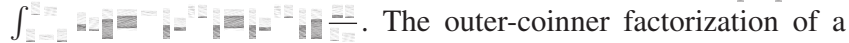
rational matrix $\ldots$ is a factorization $\equiv-$ 二 -

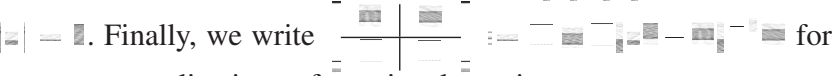
state-space realizations of a rational matrix.

\footnotetext{
${ }^{1} \mathrm{~A}$ special case is given in [8], where is is shown that if the feed-backward filter is as long as the channel and the feed-forward filter is "long enough", the optimal decision delay is the length of the forward filter minus one. Unfortunately, "long enough" in not quantified in [8].

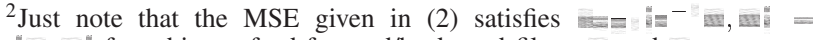

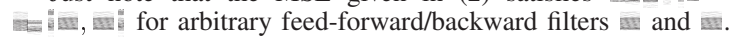




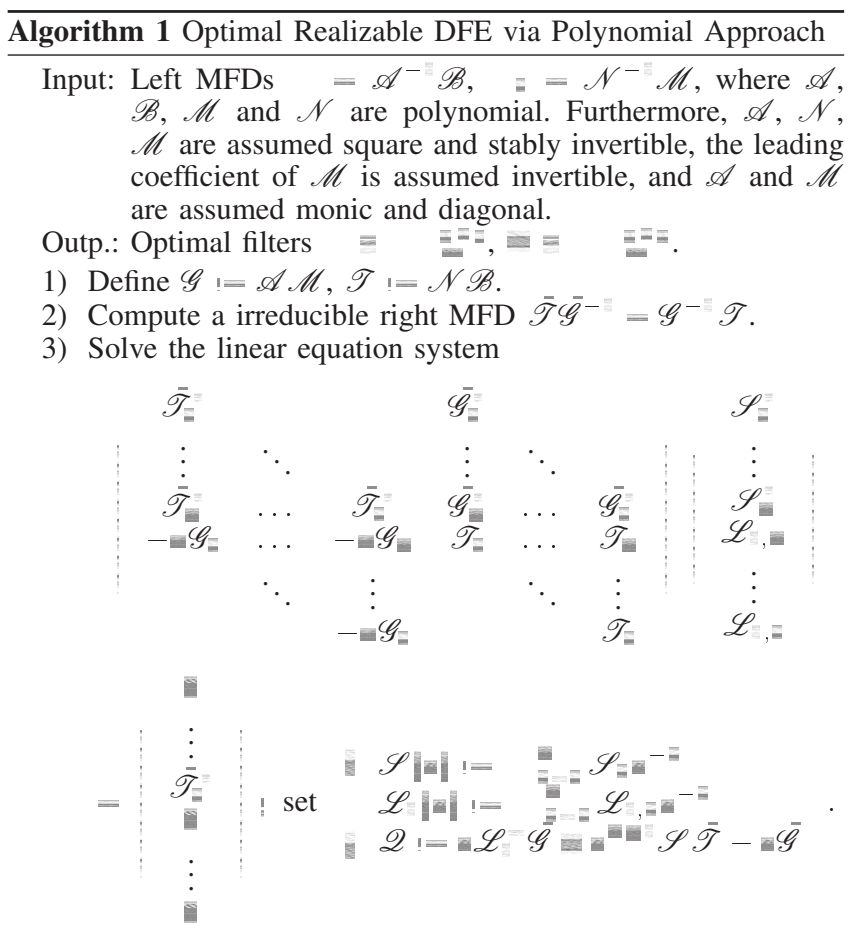

4) Return $\quad !=\mathscr{S} \mathscr{M}^{-5} \mathscr{N}, \equiv !=\mathscr{Q} \overline{\mathscr{T}}^{-!}$.

\section{SYSTEM MODEL AND PROBLEM STATEMENT}

In the $=$-domain, our system model is

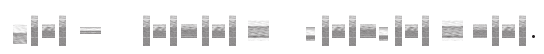

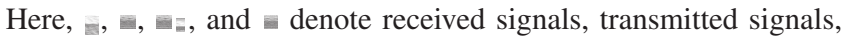
transmitted signals of an interferer, and noise. The signals a, 플, and $\equiv$ are assumed to be mutually independent, spatially and temporally white Gaussian random variables. The covariance matrices are the identity matrix for data and interference, and " - - for noise. The channels and $\equiv$ are stable and causal rational matrices, i.e., $\equiv \quad \equiv \equiv$ and $\equiv \equiv \equiv \equiv \equiv$. Our goal is to design a feed-forward filter $\equiv= \pm$ and a feed-backward filter $\equiv \equiv \equiv \equiv$, such that the (delayed) estimate ${ }^{3}$

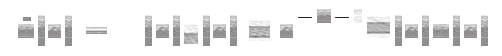

minimizes the asymptotic mean square error

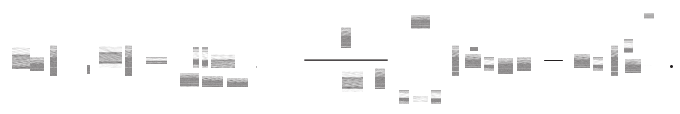

\section{POLYNOMIAL APPROACH}

The polynomial approach for optimal DFEs is due to [3]. We point out that the model in [3] is slightly different from (1). They assume ${ }^{\bar{E}}=$ and that $\equiv$ is square with invertible. However, our setting can also be recast in the setting of [3] if a spectral factor of ${ }^{-1}=$ is chosen as interferer.

We summarize the polynomial approach in Alg. 1. The authors themselves claim that the complexity grows cubically with the decision delay [3, Ch. IV.D]. A quick inspection of Alg. 1 shows

\footnotetext{
${ }^{3}$ Note that we make the usual correct past decisions assumption [1], [3],

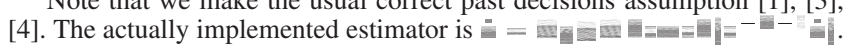

that only the steps 3) and 4) depend on the decision delay. Steps 1) and 2) are independent of the delay. The matrix in step 3) (let

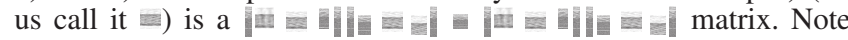
that each corner of $\mathbf{i}$ is a block-triangular Toeplitz matrix. If the channel is square (i.e., 琶= $=$ ), and we define the shift matrix

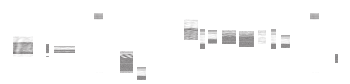

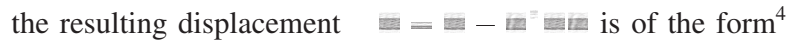

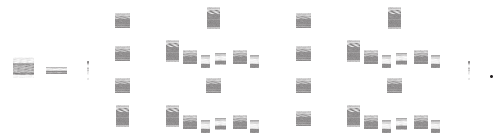

Therefore, the displacement rank of $\mathbf{a}_{\text {can }}$ be at most $\overline{\mathbf{p}}$, and efficient displacement structure based algorithms can be used to solve step 3) with complexity depending quadratically on the decision delay [10]. Computation of the polynomial matrix products in Step 3)/4) can also be achieved with quadratic complexity. Thus, the overall complexity of Alg. 1 grows quadratically with the delay.

Finally, let us point out a drawback of Alg. 1. The numerators $\mathscr{A}$ and $\mathscr{M}$ are required to commute, which is why they are chosen diagonal. However, this increases the number of coefficients in the polynomials $\mathscr{B}$ and $\mathscr{N}$. Under certain circumstances this can make step 2) of the algorithm very expensive (although overall complexity still scales quadratically with iii).

\section{INNER-OUTER FACTORIZATION APPROACH}

In principle, computation of the optimal DFE can be considered as computation of the optimal linear equalizer for a virtual MIMO channel where the feedback-path is modeled as additional noisefree part of the channel [4], [6]. In particular, the inner-outer factorization approach to linear equalization can be employed [11] However, if one naively applies linear equalization algorithms to the virtual channel, complexity grows very fast when the decision delay increases. This happens because the (McMillan) degree of the virtual MIMO channel increases with the delay which then rapidly increases complexity of the inner-outer factorization.

\section{IV-A. Derivation of the Optimal Realizable DFE}

In this section we outline how the optimal DFE can be computed in a way such that the complexity of the inner-outer factorization is independent of the decision delay. Explicit state-space formulae for implementation will be presented in the following section. We start with the observation that the MSE holds [6]

$$
\text { 或门, }
$$

Therefore the optimal filters are given by

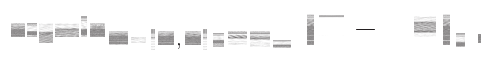

where

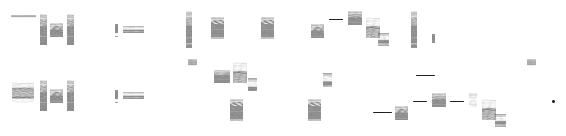

We will now determine the optimal in a way such that the complexity scales well with the decision delay in. Suppose that

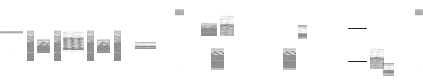

${ }^{4}$ Of course, is also highly structured if the channel is non-square. However, we haven't yet found a suitable replacement for the shift matrix ii in the non-square case such that the displacement is low rank. 
is a outer-coinner factorization ${ }^{5}$ (as defined in the notations). Then,

$$
\text { iㅔ }
$$

and (3) is equivalent to [11]

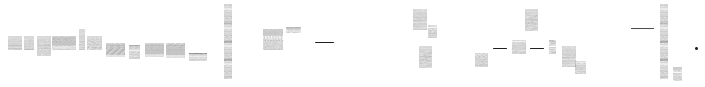

Write

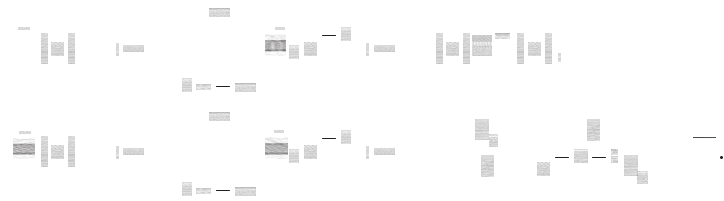

Then, Parsevals relation shows that

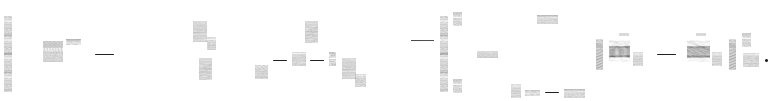

Let us partition

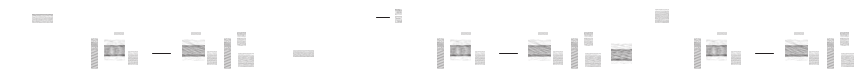

$$
\begin{aligned}
& \text { 面 }=-1 \\
& \overline{1+m=-1}=
\end{aligned}
$$

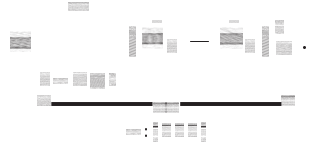

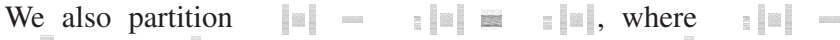
is FIR, and $\quad$ in $=-{ }^{-1}$ is. We will now successively bring each of the three sums (I)-(III) to their individual minimum.


Therefore, the sum (I) is independent of the choice of

(II) Define three matrices

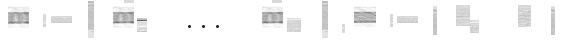

$$
\begin{aligned}
& \overline{\mathrm{E}}^{-} \mathrm{E}^{-} \quad \ldots \quad \mathrm{e}^{-}
\end{aligned}
$$

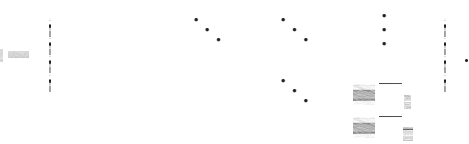

Then, we minimize the sum (II) by choosing



where $=$ is the solution to the least-squares problem

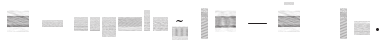

(III) Since ${ }^{1}$ is inner, we can rewrite (6) as

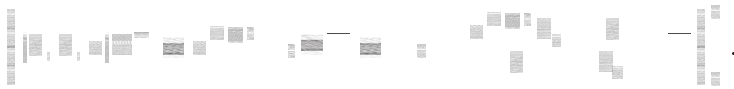

${ }^{5}$ The outer-coinner factorization is dual to the inner-outer factorization. Therefore, we still call our approach "inner-outer approach" instead of "outer-coinner approach" because the former term is more common.
Thus, with $\cdot$ being the causal part [11], does

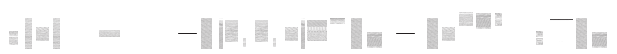

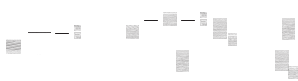

set the sum (III) to zero, which certainly is minimal Finally, note that $1 \mathrm{H}, \mathrm{E}, \mathrm{w}=\mathrm{i}$ is strictly anti-causal, and

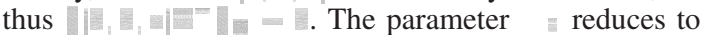

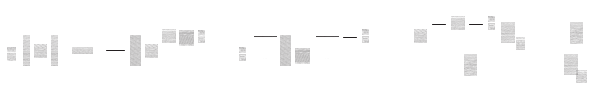

Since we have jointly reduced all three sums to their individual minimum, (7) is also minimal. Thus, (6) holds, and is an optimal parameter in (3). We summarize our findings.

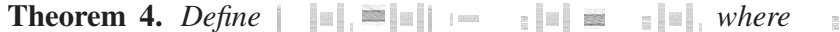
and $\equiv$ are given in (8) and (10). Then, and are optimal feed-forward and feed-backward filters.

\section{IV-B. State-Space Formulae}

Although we have now derived an efficient formula for the optimal realizable DFE, it is not clear how to actually implement it. Therefore, we will now derive state-space formulae for the optimal DFE. State-space realizations have the advantage that they allow simple evaluation in both time- and frequency-domain. Let us start with a state-space realization [12]



Explicit formulas for the outer-coinner factorization (4) are wellknown [13]. We repeat them for completeness. Let $-{ }^{m}$ denote the stabilizing solution to the discrete-time Riccati equation [14]



$$
\begin{aligned}
& \text { 일 }
\end{aligned}
$$

Then, with

$$
\begin{aligned}
& \text { 口 } \\
& \text { 回 } 1 \text { - }
\end{aligned}
$$

a state-space realization for the outer-coinner factorization (4) is

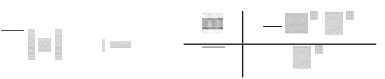

$$
\begin{aligned}
& \text { - }
\end{aligned}
$$

With these realizations, we obtain the following formulae for the entries of and,



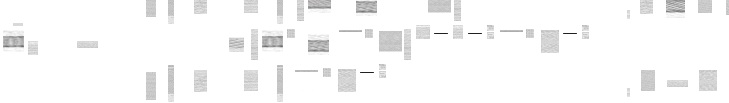

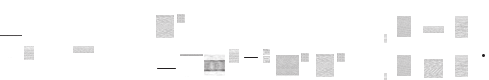

Let us now prove the following Lemma.

Lemma 5. Consider a FIR filter state-space system

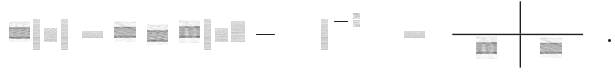




\begin{tabular}{l} 
Algorithm 2 Optimal Realizable DFE via Inner-Outer Factorization \\
\hline Input: \\
Outp.: Solution \\
1) Compute the outer-coinner factorization (11)-(12). \\
2) Compute and via (13)-(14). \\
3) Compute given in (8)-(9). \\
4) Compute given in (15). \\
5) Return
\end{tabular}

Then, with $\quad:-\quad n=\mathbb{1}$, it holds

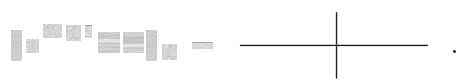

Proof: The claim follows from the identities

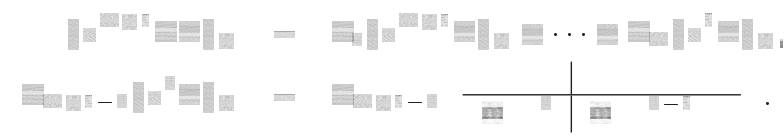

We can use the Lemma to obtain the following state-space realization of (10)

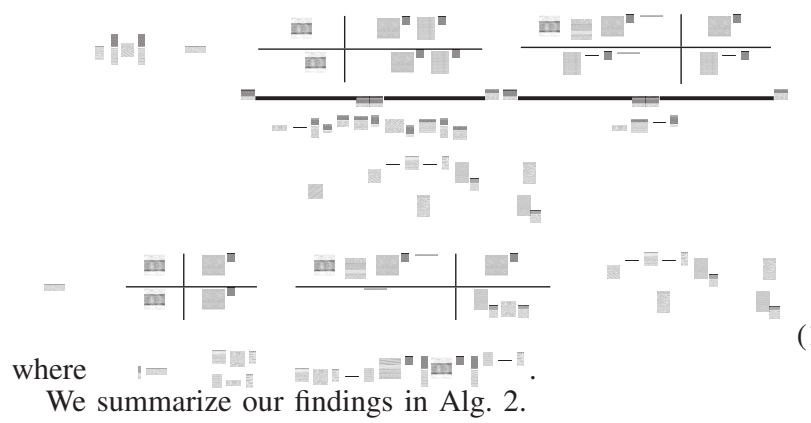

\section{IV-C. Complexity Analysis}

The complexity of Alg. 2 is as follows.

1) Step one is independent of the decision delay.

2) The complexity to compute block Toeplitz, its computation is linear in as well.

3) The matrix is a has block upper-triangular Toeplitz structure which implies a displacement rank of : Therefore, the least-squares problem (9) can be solved with complexity quadratically in [15].

4) The only thing that changes in the realization (15) (except for the trivial delay) is the matrix . The complexity of computing it scales linearly with $\boldsymbol{I}_{\text {. }}$.

\section{COMPARISON OF POLYNOMIAL AND INNER-OUTER APPROACH}

We compare Alg. 1 and 2. The polynomial approach starts with the computation of an irreducible right MFD (Step 2, see e.g. [16] for an algorithm), while the inner-outer factorization approach starts with an outer-coinner factorization (Step 1). Interestingly, both of these steps are special cases of the so-called coprime factorization [12]. The irreducible right MFD is a coprime factorization where both factors have to be polynomials, while the inner-outer factorization is a coprime factorization where the numerator matrix is inner. Note that since the numerators in Alg. 1 are required to be diagonal, the MFDs of channel and noise are not irreducible and the costs for the polynomial coprime factorization can become unnecessarily high. This issue does not appear in the outer-coinner factorization, where minimal realizations are allowed.
Then, both algorithms have to solve a set of linear equations. In the polynomial approach, the coefficient matrix is a invertible $\mathbf{s}$

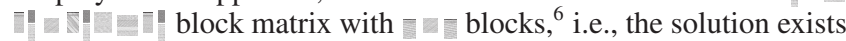
and is unique. (See [10, Ch. III] for an algorithm.) The displacement rank of the matrix is matrix is a right-invertible triangular 늘 with $-\mathbb{E}$ - blocks, and a least-squares solution is sought [15]. The displacement rank ${ }^{7}$ is smaller than in the polynomial approach. While the polynomial approach is done at this point, the inner-outer approach needs one more step (Step 4), which is the computation of grows only linearly with the delay (cf. Lemma 5).

In summary, we see that in the first two steps (coprime factorization and the least squares problem) the inner-outer approach is less complex than the polynomial approach. On the other hand, the inner-outer approach requires an additional third step (computation of complexity is negligible.

\section{REFERENCES}

[1] N. Al-Dhahir and A. H. Sayed, "The finite-length multi-input multi-output mmse-dfe," IEEE Trans. Signal Process., vol. 48, pp. 2921-2936, Oct. 2000.

[2] R. Merched and N. R. Yousef, "Fast techniques for computing finite-length mimo mmse decision feedback equalizers," IEEE Trans. Signal Process., vol. 54, pp. 701-711, Feb. 2006.

[3] C. Tidestav, A. Ahlen, and M. Sternad, "Realizable mimo decision feedback equalizers: structure and design," IEEE Trans. Signal Process., vol. 49, pp. 121-133, Jan. 2001.

[4] A. Erdogan, B. Hassibi, and T. Kailath, "Mimo decision feedback equalization from an h-infinity perspective," IEEE Trans. Signal Process., vol. 52, no. 3, pp. 734-745, 2004.

[5] B. Hassibi, A. H. Sayed, and T. Kailath, Indefinite-Quadratic Estimation and Control. Philadelphia: SIAM, 1999.

[6] S. Wahls and H. Boche, "Realizable equalizers for frequency selective mimo channels with cochannel interference," in Proc. IEEE ICASSP, (Taipei, Taiwan), Apr. 2009.

[7] P. A. Voois, I. Lee, and J. M. Cioffi, "The effect of decision delay in finite-length decision feedback equalization," IEEE Trans. Inf. Theory, vol. 42, pp. 618-621, Mar. 1996.

[8] Y. Gong and C. F. N. Cowan, "Optimum decision delay of the finite-length dfe," IEEE Signal Process. Lett., vol. 11, pp. 858-861, Nov. 2004.

[9] C. Zhang and R. R. Bitmead, "Mimo equalization with statespace channel models," IEEE Trans. Signal Process., vol. 56, pp. 5222-5231, Oct. 2008.

[10] T. Kailath and A. H. Sayed, eds., Fast Reliable Algorithms for Matrices with Structure. Philadelphia: SIAM, 1999.

[11] A. Ahlen and M. Sternad, Polynomial methods in optimal control and filtering, vol. 49 of IEE control engineering series, ch. Optimal Filtering Problems, pp. 120-161. IET, 1993.

[12] K. Zhou, J. Doyle, and K. Glover, Robust and Optimal Control. Upper Saddle River, NJ: Prentice-Hall, 1996.

[13] V. Ionescu and C. Oara, "Spectral and inner-outer factorizations for discrete-time systems," IEEE Trans. Autom. Control, vol. 41, pp. 1840-1845, Dec. 1996.

[14] V. Ionescu and M. Weiss, "On computing the stabilizing solution of the discrete-time riccati equation," Linear Algebra Appl., vol. 174, pp. 229-238, Sept. 1992.

[15] D. Kressner and P. Van Dooren, "Factorizations and linear system solvers for matrices with toeplitz structure," SLICOT Working Note 2000-2, June 2001. Revised Version.

[16] J. C. Basilio and B. Kouvaritakis, "An algorithm for coprime matrix fraction description using sylvester matrices," Linear Algebra Appl., vol. 266, pp. 107-125, Nov. 1997.

\footnotetext{
${ }^{6}$ In this section, we assume a square channel, i.e., $-=$ (cf. Sec. III).
}

${ }^{7}$ In the sense that each block is extended with zeros into a square block 